\title{
An anthology of non-local QFT and QFT on noncommutative spacetime
}

\author{
Dedicated to Detlev Buchholz on the occasion of his 60th birthday \\ Bert Schroer \\ presently: CBPF, Rua Dr. Xavier Sigaud 150 \\ 22290-180 Rio de Janeiro, Brazil \\ Prof. em., Institut für Theoretische Physik, FU-Berlin \\ email: schroer@cbpf.br
}

May 6, 2004

\begin{abstract}
Ever since the appearance of renormalization theory there have been several differently motivated attempts at non-localized (in the sense of not generated by point-like fields) relativistic particle theories, the most recent one being at QFT on non-commutative Minkowski spacetime. The often conceptually uncritical and historically forgetful contemporary approach to these problems calls for a critical review in the light of previous results on this subject.
\end{abstract}

\section{History of attempts at non-local fields}

To attribute in-depth investigations of non-local QFT to the last decade, as it is done in most contemporary articles on this subject, is historically incorrect. There was already a flurry of interest (quite a strong one considering the number of particle physicist at that time) in non-local aspects of QFT leading to significant results as far back as the late $50 \mathrm{~s}^{1}$. This interest originated in the wake of renormalization of QED and of the LSZ scattering theory. The main physical motivating idea was to get to more and "better" (in the sense of less singular) interactions by using L-invariant structure functions in the interaction part of the Lagrangian. In addition there was the desire to understand whether (pre-QCD) meson-nucleon structure, as it became experimentally accessible in nucleon formfactors, could be consistently used in a Lagrangian formalism; in other words if what nowadays would be considered as "effective interactions"

\footnotetext{
${ }^{1}$ This is of course ment relative to the much smaller number of particle physicists at that time.
} 
could be of a more fundamental significance within a non-local Lagrangian setting.

The extensive work of Kristensen and Møller [1] as well as an important contribution by Claude Bloch [2] and Hayashi [3] with prior remarks by Pauli and Rayski led to lively discussions and also called the critical attention of leading field theorists of the post renormalization era as Kallen and Lehmann [4]. The issue of non-locality attracted many Japanese researchers and became even the main topics of a 1954 conference in Japan [5]. Since relativistically invariant non-local interactions even on a formal level do not fit well with the canonical equal time formalism (and neither with the associated functional integral approach), most investigation were carried out in the Yang-Feldman setting which is independent of the canonical formulation and most closely related to both the Wightman theory and the LSZ framework of scattering theory. The main problem in this setting, which relates the interacting Heisenberg fields with on-shell in- and out- fields, is the proof that if the incoming on-shell field is assumed to have the standard free field commutation relation then this property is inherited by the on-shell outgoing field, so that the S-matrix is unitary. This is a problem which is in principle decidable in perturbation theory. The original structural argument in favor of this property in C. Bloch's work was not correct ${ }^{2}$; indeed it was noticed somewhat later [3] by explicit calculation up to fourth order of the commutator of the outgoing field within the Kristensen-Møller setting (which uses a L-invariant formfactor instead of a pointlike interaction vertex) that it is definitely violated.

Although this was the result of a particular non-local model, it was believed that this negative answer is generic to non-local interactions. A very clearly written re-investigation confirming this negative result within a more modern context appeared two decades later [6]. Since the standard derivation of the unitary S-matrix in QFT (as envisaged by Lehmann, Symanzik and Zimmermann and proven by Haag and Ruelle [7]), requires the validity of the spacelike cluster property for the correlation functions of the Heisenberg fields, a proof that the on-shell outgoing field does not fulfill the free field commutation relation shows at the same time that the cluster property is violated. The latter is part of what one summarily calls "macro-causality" 3 . Consider a partition of the field coordinates in a correlation function into two clusters which are separated in the sense that the localization points of the fields in one cluster are say to the left of those of the other cluster. Increasing the cluster distance to spacelike infinity, the vacuum expectation factorizes in those of the two clusters and the connected goes to zero with the well-known Yukawa fall-off which is given by the smallest invariant mass of the intermediate states above the vacuum between the two clusters. However if the clusters inside the expectation value are overlapping, one first has to disentangle them to the previous non-overlapping position, which requires the application of spacelike commutation relations. If

\footnotetext{
${ }^{2}$ This can easily be seen by realizing that e.g. the very existence of creation/annihilation operators fulfilling the Zamododchikov-Faddeev relations provides a counterexample.

"Strictly speaking this is "macrolocality" whereas macrocausality is more related to the absence of timelike precursors.
} 
the latter are local, there will be no modification of the fall-off, but the situation may change in case of non-local spacelike commutators.

There exists the following remarkable theorem of Borchers and Pohlmeyer [8] which for Bose fields in a theory with mass gap amounts to the following statement

Theorem 1 A spacelike fall-off of the commutator of the (Heisenberg) fields in the relative distance which is faster than Yukawa's exponential decrease leads back to a micro-causal theory.

Here faster than Yukawa means that the decrease is dominated by exp $\lambda r^{1+\varepsilon}$ with $\lambda, \varepsilon>0$ and $r$ the relative spatial distance. From this follows that the connected parts of correlation functions for genuine non-local Poincaré covariant theories with positive energy have a weaker large distance decrease than that determined by the standard intermediate state argument. In addition the clustering decrease is anisotropic in the sense that it is still Yukawa like for nonoverlapping clusters and weaker than Yukawa otherwise. Such a behavior is quite unusual in particle physics; apart from jeopardizing the standard analytic connection with the Euclidean formulation it may have visible experimental manifestations, but we will not pursue this interesting problem in this work.

This also indicates that the clustering in genuine non-local QFTs is not any more determined by the "kinematical" properties of the lowest invariant energy of intermediate states ${ }^{4}$ but rather suffers modifications which depend via the commutator fall-off on the details of the non-local interaction. In a way these results suggest that, contrary to what one may have expected intuitively and contrary to the deceivingly lighthearted manner in which computational tricks as cutoffs and regulators are sometimes interpreted as basic physical concepts, causality is an extremely rugged property and that a notion of "a little bit nonlocal" or "a little bit acausal" is not much more sensible than "a little bit pregnant". Whereas it is easy to cut-off or regularize Feynman integrals as part of perturbative renormalization theory it, is generally not known how such ideas can be consistently implemented in the operational setting of Hilbert spaces and operators.

Hence it should not come as a surprise that most attempts of introducing deviations from micro-causality actually amount to violating macro-causality and often also Lorentz invariance in the wake; but macro-causality is the absolute borderline between physics and the realm of poltergeists, and sacrificing the relativity principle without getting a conceptual benefit does not seem to hold much attraction either.

Among the more prominent attempts there was the proposal by Lee and Wick to modify the Feynman rules by pair of complex poles together with their complex conjugates. After formulating this idea in a field theoretic setting in such a way that at least the unitarity of the S-matrix within the Yang-Feldman

\footnotetext{
${ }^{4}$ The pre-exponential strength factors in local QFT do of course depend on details of the interaction.
} 
setting was maintained, Marques and Swieca [9] showed that the proposal led to physically untenable timelike power "precursors" 5 .

This raised the question whether there exist consistent relativistic unitary and macro-causal particle theories at all. A positive answer was given Coester's idea [10] of construction of "direct particle interactions". This was first formulated on 3-particle systems and then generalized (in collaboration with Polyzou) [25] to multiparticle theories. As a pure relativistic particle theory without vacuum polarization, it turns out to have no natural second quantization setting; but on the other hand it fulfills all properties which are expressible in terms of particles without inferring fields. In particular these theories fulfill the cluster separability properties of the associated Poincaré invariant unitary S-matrix; in fact even the generators of the Poincaré group cluster (though not with Yukawa like exponential decrease) as a result their existence contradicts a dictum (ascribed to S.Weinberg) saying that a Poincaré invariant unitary S-matrix which clusters is characteristic for local QFT. The possibility that this conjecture may apply to the stronger requirement of a Yukawa type clustering as known from local QFT is however not completely excluded.

Although these Coester-Polyzou (C-P) theories of direct particle interactions have acquired some popularity in phenomenological treatment ${ }^{6}$ of medium energy meson-nucleon interaction calculations (where the limitation in energy prevent the appearance of real multi-particle creation [25]), our interest in them, as far as this paper is concerned, is strictly limited to the desire to broaden our conceptual scope of the meaning of macrocausal and non-local behavior even if the objects which achieve that are strictly speaking not QFTs since, as in the case of the C-P theories, they allow no natural second quantization. As soon as one leaves the setting of local QFT, properties as vacuum polarization, TCP, spin\&statistics.. which are structural consequences of locality loose their validity, although one may in many cases add them by hand i.e. select those non-local models which have these additional properties. One should also notice that, unlike the Galilei group, the Poincaré group representations do not impose any mass superselection rule and hence one can incorporate particle creation processes into a direct particle interaction setting.

Besides these theories which, as a result of absence of a second quantization setting are not QFTs in the strict sense, no consistent non-local but macrocausal relativistic particle theories are presently known. The main subject of this paper will consists in presenting some recent attempts of how to go beyond locality in "noncommutative QFT"; more specifically how to achieve this by formulating standard QFT on noncommutative Minkowski spacetime.

With the above mentioned negative results coming from the history of nonlocal QFT from different sources, the studies about the feasibility of Lorentz invariant non-locality was laid to rest up to the beginnings of the 90's, when

\footnotetext{
${ }^{5}$ Only in the case of external interactions only the precursors have a more amenable exponential behavior.

${ }^{6}$ The work on direct particle interactions has been mainly published in nuclear physics journals which perhaps explains why it is virtually unknown in the high energy physics community.
} 
quite different well-founded physical considerations led to ideas about quantum field theory on non-commutative Minkowski spacetime. These turned out to have a more subtle relation to the issue of non-locality. The starting point was an observation by Doplicher, Fredenhagen and Roberts [14] on a quasiclassical interpretation of Einstein's general relativity equation with the added requirement that mere localization measurements (without the action of additional dynamical processes) should not generate black hole horizons.

Such a requirement was not entirely new; one finds a mentioning of localization measurements and black holes within a very restrictive (and physically somewhat less motivated) setting as far back as 1964 [15]. However a formulation of uncertainty relations allowing for localization regions of any shape, derived from a few physically plausible assumptions, really starts with the DFR work.

Nowadays one occasionally also finds references to a much older 1947 paper by Snyder [16]. This paper is sometimes quoted in a slightly wrong context since Snyder's motivating idea was to mimic lattice regularization in a covariant way, which for technical reasons led him to noncommuting spatial position operators. Without the clarification of the idea of renormalization there was anyhow no chance in 1947 to say something relevant on quantum field theoretic aspects of gravitation.

The DFR argument in favor of uncertainty relations for position operators is reminiscent of the Bohr-Rosenfeld derivation of uncertainty relations for the electromagnetic field based on a quasiclassical interpretation of electromagnetism coupled to Schroedinger quantum matter. These uncertainty relation still leave many possibilities for commuting relations between coordinate spacetime operators and DFR have chosen the simplest one. The terminology "non-commutative Minkowski spacetime" refers to the fact that even in the absence of curvature the DFR model describes a non-commutative modification of Minkowski spacetime. The Wigner's particle picture is fully incorporated in the DFR setting, but the Fourier transform which for standard free fields carries the canonical Wigner momentum space creation/annihilation operators to the spacetime dependent fields involves now operators $q_{\mu}$ obeying commutation relations which are described in terms of an antisymmetric matrix $Q$ with variable center-valued entries.

It is not clear how the original motivation of DFR of reconciling general relativity with quantum physics can be implemented since the uncertainty relations as well as the commutation relations have not been reconciled with the property of general covariance and background independence. Therefore the DFR non-commutative setting is presently limited to explore the concept of QFT on non-commutative Minkowski spacetime.

The novel feature of QFT on non-commutative spacetime is that in addition to the Wigner multi-particle momentum Fock space there is an additional Hilbert space of wave functions which describes the localization aspects in $q$ space and on which the Lorentz transformations also act. Picking a family of localizing wave functions, e.g. the family which belongs to minimal localization (which cannot be pointlike in all 4 components as a result of the uncertainty 
relations [14]) and forgetting that these wave functions (unlike test functions) suffer an active change under L-transformations as, this setting may appears like a modern form of the pre-Einsteinian "ether" 7 . But in a setting where the center-valued $Q$ remain dynamical variables in an altogether Poincaré covariant representation space, this historical analogy may be is misleading.

What seems to be implied in the DFR setting is a radical paradigm change of the meaning of "relativity principle" and "inertial systems" in a direction of which the conceptual basis has not been completely understood. Only if for some reason we were to work in an irreducible representation of the coordinate operators (in which their commutators obey necessarily non-L-invariant numerical valued commutations relations) and the Poincaré group ceases to have an automorphic action on the algebra, this would indeed amount to a return of a kind of (quantum) ether. One also should refrain from interpreting the $q^{\prime} s$ as some kind of quantum mechanical observables (the terminology "uncertainty relations" in [14] may invite such an interpretation) as e.g. a quantum mechanical Newton-Wigner [17] localization operator ${ }^{8}$.

The conceptual problems posed by such an unusual situation of a QFT on noncommutative Minkowski spacetime are best discussed in the setting of free fields where no problems are encountered (see later). However there are serious difficulties with interactions. Intuitively one would expect that the noncommutative Minkoski space is an arena for quantum fields which for large distances presents itself as the ordinary Minkowski spacetime. Since on-shell quantities like the S-matrix are not directly dependent on short distance properties (but only indirectly via the encoding into long distances via particle scattering data) one would think that the very concepts of incoming and outgoing (Wigner) multi-particle states are remaining intact. However, as will be seen later, there are serious problems with these expectations about scattering concepts.

There is a lack of standard covariance, although a less radical one, in the already mentioned "direct particle interaction" models since the requirement of additivity in particle interactions and the cluster requirement cannot be met simultaneously in one formula for the Lorentz generators. As in the case of the DFR setting, the physics of the C-P theory depends on additional data (on localization wave functions in the case of the D-F-R model, the choice of the scattering equivalence in the $\mathrm{C}-\mathrm{P}$ setting). This to be expected because the D-F-R noncommutative localization and the C-P implementation of cluster properties depend on additional choices.

Starting from some observations about the role of noncommutative tori in string theory, there has been a different line of thought leading to noncommutative and explicitly non-L-invariant theories. Such attempts are of course

\footnotetext{
${ }^{7}$ Indeed in most treatments the center-valued $Q$ are assigned fixed numerical values and hence the relativity principle in form of the 10-parametric family of inertial systems is violated in terms of the return of an ether.

${ }^{8}$ Although the asymptotically covariant Newton-Wigner localization does not play any direct role in the formulation of QFT on non-commutative Minkowski spacetime, it is crucial (as for any relativistic particle theory) in the formulation of the asymptotic behaviour which leads to the scattering operator.
} 
expected to carry all the conceptual fragility of string theory, which employs a lot of advanced mathematical techniques but tends to be atrophic on the side of physical concept and physical interpretations. But even if a strong commitment to history and faithfulness to the principles underlying particle physics would make it difficult to accept arguments from string theory at face value, there always remains the possibility to critically analyze them (independent of their origin) in the light of the conceptual framework of QFT. This will be the strategy in the rest of this section.

There exist several papers in which (motivated by string theory) the authors propose numerical-valued commutators between spacetime coordinates which break Lorentz invariance down to a subgroup. For example in [18] the subgroup of $P(4)$ turns out to be $P(2) \otimes E(2)$ i.e. tensor product symmetry of a twodimensional $P(2)$ Poincaré- with the two-dimensional Euclidean- group $E(2)$. The associated locality is that of a $1+1$ dimensional QFT of the $P(2)$ factor, whereas the $E(2)$ Euclidean degrees of freedom are just "spectators" as far as locality is concerned.

As a logical consequence of this assumption and the well-known fact that causality imposes a Lorentz invariant shape on the associated positive energy momentum spectrum [7], one finds that so that the $1+3$-dimensional Wigner particle structure gets lost and with it the relevance of such a model for $1+3$ dimensional particle physics. As a 1+1-dimensional local theory it naturally fulfills the associated TCP theorem, which combined with the reflection invariance of the Euclidean spectator theory then leads to a higher dimensional TCP operation $^{9}$. In this connection one should recall that QFT, different from QM, is conceptually intrinsic. In other words something which smells, trumpets and appears like an elephant really is an elephant.

Apparently the authors in [19] tried to address these problems. They propose a theory which maintains the usual particle concept associated with the mass hyperboloids, but breaks L-invariance through the application of a Moyal starfunctor to the Wightman functions of an interacting local Poincaré-invariant QFT (see equ. II.3 in [19], the mathematical difficulties in attributing a precise meaning are not discussed there). They show that if one lets this functor also change the commutator to a kind of spacelike star-commutativity, the weak locality assures that the old TCP operator and the S-matrix remain unchanged under the action of the star functor. If instead one were to change the local action by a star-product prescription, none of structural properties as TCP, spin\&statistics, Jost-Lehmann-Dyson representation etc. is expected to survive.

The star-functor (as any star functor belonging to a fixed set of numericalvalued $q$-commutators) does not commute with the Lorentz boosts and therefore "breaks" L-invariance but without harming the Wigner mass shell support of particles; in fact it preserves precisely the aforementioned subgroup and results in the D-F-R setting from choosing minimally localizing wave functions on noncommutative Minkowski spacetime (which lead precisely to the numerical

\footnotetext{
${ }^{9}$ In the same vein the analytic spacetime properties of the model are fixed by the BargmannHall-Wightman theorem [43] in $\mathrm{d}=1+1$.
} 
values of $Q_{\mu \nu}$ used in [19] and other papers quoted therein). But in order to attribute meaning to this words one should give an intrinsic physical definition under what circumstances a mathematical subgroup can be called a "broken symmetry" of the ambient group. Aside from the well-defined notion of spontaneous symmetry breaking (this happens to internal symmetry groups in the Goldstone setting and to the Poincaré group for a QFT in a thermal KMS state where the boosts fail to be implementable), I am not aware of such a meaning. It seems to me the acceptance of such a terminology as a part of particle physics would indeed amount to a return of the pre-Einsteinian non-dynamic ether in the setting of quantum physics.

In regards to the $P(2) \otimes E(2)$ symmetry, there is an interesting analogy with the 7-parametric subgroup of the Poincaré group which features as the symmetry group of the lightfront and which contains the 4-parametric $P(2) \otimes E(2)$ in a natural way [20]. The difference is that in the process of holographic lightfront projection the spacetime interpretation changes radically in accordance with the fact that the lightfront is not a hyperbolic manifold. This change is necessary in order to be able to return to the Poincaré covariant ambient theory. In this case the fact that the symmetry is reduced has a clear-cut physical meaning in terms of the holographic spacetime encoding whereas in the interpretation of $P(2) \otimes E(2)$ within a noncommutative setting the broken symmetry support has no physical meaning; in particular the Poincaré symmetry $P(4)$ does not return for large distances.

There is of course also the general feeling that one should not be able to generate a new physical reality by doing nothing else than applying a functor to an existing theory. In this context it is helpful to remind oneself that the Heisenberg (commutator-) ring is non-isomorphic to the classical Poisson ring, i.e. the transition from classical to quantum physics is "artistic" and not functorial (remember Nelson's famous saying: second quantization is a functor but first quantization remains a mystery).

The only way to counteract the return to a (quantum) "ether" however is to find an extension in which that $P(2) \otimes E(2)$ symmetric theory becomes part of a larger Poincaré-invariant setting. This is precisely what the D-F-R theory [21][22][23] achieves; it does so by building a QFT over a non-commutative version of fully Poincaré-invariant Minkowski spacetime. In a more colorful artistic terminology one may say that the static ether is rendered dynamic i.e. by not simply combining the possible numerical values of $Q_{\mu \nu}$ into a direct integral or fibre bundle, but semantic wrappings insinuating analogs can be no substitute for a yet incompletely understood conceptual situation.

Interpreting the word "non-local" in its widest sense as referring to any theory whose content cannot be expressed in terms of pointlike fields ${ }^{10}$, the recently discovered string localized fields also feature under the heading "non-local" in this article. By string-localized fields we mean fields $A(x, e)$ as they arose in connection with constructing the field theory behind the massless infinite spin

\footnotetext{
${ }^{10}$ In the terminology of AQFT any theory whose net of algebras cannot be generated by additivity from arbitrarily small double cone localized algebras.
} 
(rather infinite helicity tower) Wigner representation of the Poincaré-group [11]. Here $x$ denotes a point in Minkowski space and $e$ is a "fluctuating spacelike direction" i.e. a localization point of a quantum field in 3-dimensional de Sitter space. Such strings are causal if their spacelike half-lines $x+R_{+} e$ are relatively spacelike (not just their endpoints). There are very good reasons to believe that there exist interactions of string-localized fields which maintain the string localization in each order. There also exist semiinfinite string-localized massive free fields. Even though they generate the same Wigner one-particle spaces as their pointlike counterparts, there are good reasons to believe that they widen the concept of particle interactions while maintaining appropriately adjusted causal localization properties [45].

Finally it is worthwhile to point out that non-local objects play a crucial intermediate auxiliary role in the nonperturbative construction of local theories. A well-known example is supplied by the vacuum-polarization-free-generators (PFGs) of $\mathrm{d}=1+1$ factorizing models whose Fourier transforms are momentum space creation/annihilation operators which fulfill the Zamolodchikov-Faddeev algebra relations and are therefore non-local. They turn out to be wedgelocalized; in fact they generate the wedge algebra. They do not have the form of a smeared pointlike localized field with the wedge being the support of the smearing function; rather they are linked in an inexorable way to the global wedge region [13]. The only way to get to sharper localized subwedge algebras is by intersecting wedge algebras. In this process the generators change; they acquire vacuum polarization and are represented in terms of infinite series in the Z-F operators.

These ideas about non-local generators of wedge algebras are important, because they show that one of the motivating pillars for studying non-local theories namely the idea that local theories are inherently beset by ultraviolet problems is not true. What causes these problems is not the local structure of QFT but rather the use of "pointlike field coordinatization" in the calculational approach. Whereas the use of problem-creating singular coordinates in geometry can be avoided, the "coordinates" of standard QFT obtained via Lagrangian quantization in the form of fields are inherently singular. This is of course the main reason why the algebraic approach which is based on nets of (bounded operator) algebras was proposed instead of the standard pointlike field formulation. There are myriads of (composite) fields which all generate the same net of algebras. In a way the spacetime indexed net of algebras corresponds to local equivalence classes of fields (Borchers classes) and not to individual pointlike fields. Hence the motivation for using non-local operators, either as auxiliary tools in local theories or for the construction of inherently non-local theories is rather similar, in both cases one wants to avoid ultraviolet problems.

In our presentation we avoid anything which could be understood as an axiomatic formulation. We think that the scarcity of controllable examples and the poor mathematical and conceptual status presently does not warrant an axiomatic approach to non-locality and non-commutativity.

The content is organized in the following way.

In the next section we present the relativistic direct particle interaction 
setting which fulfills cluster factorization and the time like aspects of macrocausality (absence of precursors). In section 3 semiinfinite string-localized fields are introduced, whereas section 4 shows the usefulness of non-local (in fact wedge-localized) operators in the nonperturbative construction of local theories. Finally section 5 presents details about QFT on noncommutative Minkowski spacetime and its encoding into non-local QFT on standard Minkowski spacetime. Some open problems are mentioned in the last section.

\section{Direct particle interactions and macro-causality}

It has been known since the early days of particle physics that an interacting relativistic 2-particle system of massive particles (for simplicity of equal mass) is simply described by going into the c.m. system and modifying the mass operator in the following way

$$
\begin{aligned}
& M=2 \sqrt{\vec{p}^{2}+m^{2}}+v \\
& H=\sqrt{\vec{P}^{2}+M^{2}}
\end{aligned}
$$

The interaction $v$ may be taken as a function of the relative coordinate which is conjugate to the relative momentum $p$ in the c.m. system; but since the scheme does not lead to local differential equations, there is nothing to be gained to insist in such a locality property. One may the follow Bakamjian and Thomas (BT) [24] and choose the $\vec{P}$-generators in such a way the interaction does not appear $^{11}$ in the formulas for the c.m. momentum $\vec{P}=\vec{P}_{0}$, the total angular momentum $\vec{J}=\vec{J}_{0}$, as well as in the position conjugate to the c.m. momentum $\vec{X}=\vec{X}_{0}$, whereas the boost generators depend implicitly on the interaction through

$$
\vec{K}=\frac{1}{2}\left(H X_{0}+X_{0} H\right)-\vec{j} \times \vec{P}(M+H)^{-1}
$$

The Wigner canonical spin $\vec{j}=\vec{J}_{0}-\vec{X}_{0} \times \vec{P}$ commutes with $\vec{X}_{0}$ and $\vec{P}$ and results from applying the boost transformation $L_{P}$ on the Pauli-Lubanski vector $W$

$$
\begin{aligned}
L_{P} W & =(1,0,0, M \vec{j}) \\
W_{\mu} & =\frac{1}{2} \sum_{\nu \rho \sigma} J^{\rho \sigma} P^{\nu} \varepsilon_{\nu \rho \sigma \mu}
\end{aligned}
$$

It is now easy to check that the commutation relations of the Poincaré generators are a result of the above definitions and the canonical commutation relations of the single particle canonical variable which furnish a complete (irreducible) set of operators in terms of which any operator in the Hilbert space may be

\footnotetext{
${ }^{11}$ The group theory alone does not select a particular way of introducing interactions; in fact the C-P scheme uses this flexibility in the multiparticle sectors for the implementation of the all important cluster properties.
} 
written. Furthermore for short ranged interactions $v$ the sequence of unitaries $e^{i H t} e^{. i H_{0} t}$ converges strongly towards the isometric Møller operators [10], from which in turn one may compute the S-matrix

$$
\begin{aligned}
\Omega_{ \pm}\left(H, H_{0}\right) & =s-\lim _{t \rightarrow \pm \infty} e^{i H t} e^{-i H_{0} t} \\
S & =\Omega_{+}^{*} \Omega_{-}
\end{aligned}
$$

The unitarity of $S$ in the subspace orthogonal to the possible bound states is a consequence of the identity of ranges of $\Omega_{ \pm}$. Since the Hamiltonian is framedependent, and a relativistic particle theory is only physically acceptable if the scattering operators are frame-independent, we must have

$$
\Omega_{ \pm}\left(H, H_{0}\right)=\Omega_{ \pm}\left(M, M_{0}\right)
$$

which indeed follows from the same kind of short range assumptions [10] which already assured the validity of the asymptotic convergence. The same short range is also responsible for the cluster property, namely the statement that the infinite translation of one particle to spatial infinity results in the S-matrix converging against the identity (the product of the single particle S-matrices) $\Omega_{ \pm} \rightarrow 1$.

The BT form of the generators can be achieved for arbitrary number of particles. As will be seen, the advantage of this form of the representation is that in passing from n-1 to n-particles the interactions simply add and one ends up with Poincaré group generators for an interacting n-particle system. But whereas this iterative construction in the nonrelativistic setting complies with cluster separability, this is not the case in the relativistic context. This problem shows up for the first time in the presence of 3 particles [10]. The BT iteration from 2 to 3 particles gives the 3 -particle mass operator

$$
\begin{aligned}
M & =M_{0}+V_{12}+V_{13}+V_{23}+V_{123} \\
V_{i j} & =M(i j, k)-M_{0} \\
M(12,3) & =\sqrt{\vec{p}_{12,3}^{2}+M_{12}^{2}}+\sqrt{\vec{p}_{12,3}^{2}+m^{2}}
\end{aligned}
$$

Here $M(12,3)$ denotes the 3 -particle invariant mass in case the third particle is a "spectator" which does not interact with 1 and 2 . The momentum in the last line is the relative momentum between the (12)-cluster and particle 3 in the joint c.m. system i.e. $\vec{p}_{12,3}=L_{P}^{-1}\left(\vec{p}_{12}-\vec{p}_{3}\right), \vec{p}_{12}=p_{1}+p_{2}$. As in the nonrelativistic case one can always add a totally connected contribution. This generally changes the S-matrix of the full system while keeping the lower particle S-matrices. But contrary to the nonrelativistic case the BT generators constructed with $M$ do not fulfill the cluster separability requirement. The latter demands that if e.g. the interaction between two clusters is removed, the unitary representation factorizes into that of the product of the two clusters. Applied to the case of 3 particles with particle 3 being a spectator, one expects that shifting the third particle to infinity will result in a factorization $U_{12,3}(\Lambda) \rightarrow U_{12}(\Lambda) \otimes U_{3}(\Lambda)$. But 
what really happens $[26]$ in the limit is

$$
U_{12,3}(\Lambda) \rightarrow U_{1}(\Lambda) \otimes U_{2}(\Lambda) \otimes U_{3}(\Lambda)
$$

The reason for this violation of the cluster separability property (as a simple calculation using the transformation formula from c.m. variables to the original $p_{i}, i=1,2,3$ shows) is that the translation in the original system (instead of the c.m. system) does remove the third particle to infinity but it also drives the two-particle mass operator (with which it does not commute) towards its free value (for an explicit calculation of this limit see [26]). The BT construction is very well suited to manufacture a Poincaré covariant 3-particle interaction which is additive (6) in the respective c.m. interaction terms, but the $U(\Lambda)$ of the resulting system will not be cluster-separable in the sense of the previously two-particle system.

Fortunately there always exist unitaries which transform BT systems into cluster-separable systems without affecting the S-matrix. Such transformations are called scattering equivalences. The phenomenon behind this observation is vaguely reminiscent of the insensitivity of the S-matrix in QFT against local changes in the interpolating field-coordinatizations which in field theoretic terminology means changing the pointlike field by passing to another (composite) field in the same Borchers class, or in the setting of AQFT by picking another operator from a local operator algebra ${ }^{12}$. The notion of scattering equivalences is conveniently described in terms of a subalgebra of asymptotically constant operators $C$ defined by

$$
\lim _{t \rightarrow \pm \infty} C^{\#} e^{i t H_{0}} \psi=0
$$

where $C^{\#}$ stands for both $C$ and $C^{*}$. These operators which vanish on dissipating free wave packets in configuration space form a ${ }^{*}$-algebra which extends naturally to a $\mathrm{C}^{*}$-algebra $\mathcal{C}$. A scattering equivalence is a unitary member $V$ of $\mathcal{C}$ which is asymptotically equal to the identity

$$
\lim _{t \rightarrow \pm \infty}\left(V^{\#}-1\right) e^{i t H_{0}} \psi=0
$$

The relation to scattering theory comes about through the change of the Møller operators according to $\Omega_{ \pm}\left(V H V^{*}, V H_{0}\right)=V \Omega_{ \pm}\left(H, H_{0}\right)$ which leaves the Smatrix unchanged. Scattering equivalences do however change the interacting representations of the Poincaré group $U(\Lambda, a) \rightarrow V U(\Lambda, a) V^{*}$

It has been shown by Sokolov [27] that these scattering equivalences can be utilized for achieving cluster separability of the (interacting) representation of the Lorentz group while maintaining the S-matrix. For example if we were to take the BT representation for the full 3-particle mass operator (6) then there exists a unitary $B$ which does the following

$$
H_{c l}=B H_{B T} B^{*}
$$

\footnotetext{
${ }^{12}$ The class of fields which interpolate the same S-matrix is much larger (it comprises all "allmost local" fields [7]) but in a local theory there is no demand for such generality.
} 
where $H_{c l}$ denotes the Hamiltonian associated with the clustering representation. In general these B's have a complicated (non-algebraic) functional analytic dependence on the interaction data in $H_{B T}$ since scattering theory enters in their calculation in an essential way. The simplest case for an explicit construction is the above mentioned case of 3-particles with one spectator. which also serves as simple demonstration of the failure of clustering in the BT setting [26].

For a consistent formulation including bound states involving more than two interacting particles, one needs the theory of rearrangement collisions which uses in addition to the Hilbert space of the interacting system $\mathcal{H}$ an auxiliary Hilbert space $\mathcal{H}_{f}$ describing the free moving stable fragments (elementary and bound particles). Since the Hamiltonian of the free dissipating fragments and the actual Heisenberg Hamiltonian operate in different Hilbert spaces we need an isometric map $\Phi$ which relates the two in such a way that the Møller operators

$$
s-\lim _{t \rightarrow \pm} e^{i t H} \Phi e^{-i t H_{f}}=\Omega_{ \pm}\left(H, \Phi, H_{f}\right)
$$

are now isometries with the same ranges between the two spaces and $S=$ $\Omega_{+}\left(H, \Phi, H_{f}\right)^{*} \Omega_{-}\left(H, \Phi, H_{f}\right)$ is a unitary operator in $\mathcal{H}_{f}$. In case of absence of bound states (or for two particles after projecting onto the subspace of scattering states) one may choose $\Phi=1$ and the formalism reduces to that in one Hilbert space $\mathcal{H}=\mathcal{H}_{f}$. Although there is a lot of freedom in the choice of $\Phi$, some knowledge about bound state wave function is necessary.

The proof that a clustering Poincaré invariant and unitary S-matix exists (for a given 2-particle interaction) is inductive [25]. The induction starts with two particles as above, where the BT additivity and the cluster property hold simultaneously. As stated above, the cluster property does not hold for 3 particles with one particle in a spectator position. The induction assumption is

- For each proper subsystem $\mathcal{C}_{i}$ of an N-body system there is a representation $U_{c l, \mathcal{C}_{i}}(\Lambda, a)$ which clusters and is scattering equivalent to a BT representation $U_{c l, \mathcal{C}_{i}}(\Lambda, a)=B_{\mathcal{C}_{i}} U_{B T, \mathcal{C}_{i}}(\Lambda, a) B_{\mathcal{C}_{i}}^{*}$ (remember the BT system has a mass operator $M_{B T, \mathcal{C}_{i}}$ which commutes with the $\vec{X}_{0}, \vec{J}_{0}, \vec{P}$ associated with $\left.\mathcal{C}_{i}\right)$

- Let $\mathcal{P}=\cup_{i} \mathcal{C}_{i}$ be a partitioning of the N-body system into proper subsystems and let $U_{\mathcal{P}}(\Lambda, a)=\prod_{i} U_{c l, \mathcal{C}_{i}}(\Lambda, a)$ a representation of the Poincaré group associated with the cluster partition $\mathcal{P}$.

Theorem 2 (Coester-Polyzou, [25][28]) $U_{\mathcal{P}}(\Lambda, a)$ is scattering equivalent to a $B T$ system $U_{B T, \mathcal{P}}(\Lambda, a)$ corresponding to a mass (invariant energy) operator $M_{B T, \mathcal{P}}$

The last step consists in writing down the BT mass operator for the full $\mathrm{N}$-body system in terms of an additive formula which is the $\mathrm{N}$-particle analog of (6) and then constructing another scattering equivalence $B$ which transforms 
this N-body BT system into a system which clusters for any partitioning

$$
s-\lim _{\left\|a_{i}\right\| \rightarrow \infty}\left(U_{c l}(\Lambda, a)-\prod_{i} U_{c l, \mathcal{C}_{i}}(\Lambda, a)\right) e^{i \sum_{i} P_{\mathcal{C}_{i}} a_{i}}=0
$$

where the last factor denotes the translation which shifts the clusters of the chosen clustering to infinity $\left(P_{\mathcal{C}_{i}}\right.$ is the momentum of the $\mathrm{i}^{\text {th }}$ cluster). The induction starts at $\mathrm{N}=2$ where all the properties are obviously fulfilled.

The direct particle interaction setting of Coester and Polyzou incorporates all those aspects of particle physics which can be formulated without local fields. Micro-causality, locality, the crossing property of the S-matrix do not belong to these properties, whereas Poincaré-invariance and the cluster separability of the scattering operator are naturally incorporated. In addition there are a number of properties which are compatible but cannot be derived as structural consequences in this setting as: existence of antiparticles, TCP symmetry, spin and statistics ${ }^{13}$. The direct particle interaction theory has been included in this anthology of non-local particle theories, because the assumptions on which it is founded define a catalogue of properties which are indispensable in any theory of relativistic particles, local or non-local.

It is worthwhile to mention that Dirac's hole model of particles/antiparticles interacting with a quantized electromagnetic field (which led to many low order correct perturbative results, see Heitler's book) despite its undeniable successes is not a correct theory of interacting particles and fields; this is the reason why it was not possible to do renormalization theory in the setting of hole theory. The reason is that (apart from some $\mathrm{d}=1+1$ integrable models) the processes of filling the "sea" and switching on the interaction do not commute, although in graphs without loops (vacuum polarizations) are not affected. The C-P direct particle interaction theory is the only known consistent relativistic particle framework which avoids the use of quantum fields and maintains consistency on the level of particles. It is non-local because the only notion of localization which (as in nonrelativistic theory) can be expressed in terms of localizing projectors (the Newton-Wigner localization) is only asymptotically L-covariant and quasi-local. But this is enough to obtain a L-invariant and clustering S-matrix. In fact Wigner particles only enter QFT through the LSZ asymptotics; the limitation of localization resulting from the non-existence of finitely localized covariant projection operators does not cause harm to covariance of scattering theory.

The description of finite propagation, as distinguished from asymptotic scattering, requires a different localization which is inherent in QFT, but has also a well-defined intrinsic meaning on particle states (related to the modular localization in section 4). It does not play a role in the Coester-Polyzou setting which is basically a scattering theory of relativistic particles and not a description of propagation over finite spacetime separations. An antagonism as pronounced as expressed in the title "Reeh-Schlieder defeats Newton-Wigner" [30] does not

\footnotetext{
${ }^{13}$ According to Kuckert [29] a spin statistics connection can be derived in the setting of nonrelativistic QM which possibly lacks a second quantization formulation. This suggsts the interesting question whether these kind of arguments can be carried over to the CoesterPolyzou direct relativistic particle interaction setting.
} 
really exist. Instead of emphasizing the lack of covariance and causal localizability of localization concepts build on the existence of projectors onto subspaces [31] and in particular of the N-W particle localization, it would have been more helpful for particle physics to emphasize that they are asymptotically covariant and causal and that they are indispensable for the derivation of scattering theory were only the asymptotic localization properties are relevant. The statement that their use in propagation over finite times creates contradictions to Fermi's two-atom Gedankenexperiment (designed to illustrate the validity of the maximal velocity principle in the relativistic quantum realm [32]) is quite irrelevant; the causal propagation aspects are to be described by the modular localization which leads to projectionless expectation values [33][34].

It is also important to note that scattering theory and asymptotic completeness lead to the description of the Hilbert space of the theory in terms of a Fock space of multi-particle states. Whereas field coordinatizations are (as coordinates in geometry) highly arbitrary, particles belong to the intrinsic and unique content of particles physics.

QFTs as those one encounters in generic curved spacetimes, lack this powerful particle aspect; they even possess no vacuum state. A similar loss of particle structure is encountered if one passes from a ground state to a thermal situation by placing the system into a heat bath [35]; instead of Wigner particles, one confronts an ensemble of dissipating quanta which do not comply with scattering theory nor relate to a Fock space structure. This situation also prevails if the thermal aspect is generated by localization via a causal horizon as in the Hawking-Unruh effect. There is simply no LSZ scattering behavior and no Fock space structure associated with Unruh excitations ${ }^{14}$. The hallmark of an Hawking-Unruh situation, as compared to a generic heat bath caused thermality, is that there exists a "territorial" extension [36][37] beyond "horizons" which is accompanied by an extension of the type $\mathrm{III}_{1}$ thermal von Neumann algebra with a KMS state at the Hawking-Unruh temperature to a standard type I ground state algebra with a Wigner particle structure and an associated scattering theory which permits to describe the Hilbert space of the theory in terms of a Fock space of incoming particles; this extension is only possible at that particular temperature. Note that our use of the notion of (Wigner) particles, as those objects in terms of which all field states above the ground state can be asymptotically resolved, is more restrictive that in [38][39].

\section{String-localized fields, stability under pertur- bations}

The simplest illustration of string-localization results from the association of a localized quantum field to the famous class of Wigner's zero mass representation of the Poincaré group for which the stability group (the "little group") of

\footnotetext{
${ }^{14}$ The quantization of an interaction-free theory on Rindler spacetime of course has the Fock space structure of the free quanta, but without the validity of the LSZ scattering theory in a Rindler world this does not extend to the interacting case.
} 
a lightlike vector has a faithful representation. Instead of one helicity, as in the conformally invariant cases of photons and massless neutrinos, this representation contains a tower of all integer- or halfinteger-valued helicity degrees of freedom of both signs and we will therefore refer to this class of representations (which depends on one continuous parameter $\kappa$, a kind of euclidean mass) as the helicity tower or "infinite" spin representation [11].

It had been known for some time that there can be no pointlike covariant field which applied once to the vacuum leads to a one-field subspace containing such a representation [40]. In more recent times, the application of the spatial version of modular theory applied to positive energy representation via forming intersections of wedge-localized subspaces in positive energy Wigner representations has led to a theorem that they are always localizable in (arbitrary thin) spacelike cones [41]. Since the cores of such cones are semiinfinite linear spacelike strings (in analogy to points being the cores of compact double cone), it is natural to look for a string-localized field as being the best (tightest localized) field theoretic description of Wigner's zero mass infinite helicity tower representations. This object indeed exists and in the following we will describe this construction in some detail.

The irreducible zero mass, infinite helicity-tower representations of the orthochronous proper Poincaré group $\mathcal{P}_{+}^{\uparrow}$ are induced from unitary irreducible representations of its stabilizer subgroup of a fixed light-like vector (the "little group"). The stabilizer group in this case is isomorphic to the two-dimensional Euclidean group $E(2)$, consisting of rotations $R_{\vartheta}$ by an angle $\vartheta \in \mathbb{R} \bmod 2 \pi$ and translations by $c \in \mathbb{R}^{2}$. Let $\varphi_{1} \cdot \varphi_{2}=\int \delta\left(|k|^{2}-\kappa^{2}\right) \overline{\varphi_{1}(k)} \varphi_{2}(k)$ (where the bar denotes complex conjugation) be the scalar product on the Hilbert space $H_{\kappa}$ of functions on the plane, restricted to the circle of radius $\kappa$. An irreducible unitary action of $E(2)$ on $H_{\kappa}$, with the Pauli-Lubanski parameter $\kappa$, which is the Casimir invariant labelling nonequivalent representations, is given by the formula

$$
\left(D_{\kappa}\left(R_{\vartheta}, c\right) \varphi\right)(k)=e^{i c k} \varphi\left(R_{\vartheta}^{-1} k\right)
$$

where $\left(R_{\vartheta}, c\right) \in E(2)$ are the Euclidean rotation and translation and $\kappa$ is a kind of Euclidean mass. The representation can be linearized by Fourier transformation with respect to $\mathrm{k}$.

Let $\psi(p)$ be an $H_{\kappa}$-valued wave function of $p \in \mathbb{R}^{4}$, square integrable with respect to the Lorentz invariant measure $d \mu(p)=\theta\left(p^{0}\right) \delta\left(p^{2}\right)$ on the mantle $\partial V^{+}$ of the forward light cone $V^{+}$. The unitary Wigner transformation law for such a wave function reads

$$
U(a, \Lambda) \psi(p, k)=e^{i p a} D_{\kappa}(R(\Lambda, p)) \psi\left(\Lambda^{-1} p\right)
$$

where $R(\Lambda, p)=B_{p}^{-1} \Lambda B_{\Lambda^{-1} p} \in E(2)$ denotes the Wigner "rotation" with $B_{p}$ an appropriately chosen family of Lorentz transformations that transform the standard vector $p=(1,0,0,1)$ to $p \in \partial V^{+}$. The Fourier series decomposition of $k \in S_{\kappa}^{1}$ leads to the discrete integer-valued helicities. Different from the zero mass finite spin representations, the occurrence of the opposite helicity in the infinite helicity tower makes a doubling of the representation (in order to 
achieve TCP invariance) unnecessary. A valuable hint as to how to investigate the localization aspects of this representation comes from placing it into the setting of tensor product representations of the form

$$
U^{0}(\Lambda, a) \otimes U^{1}(\Lambda)
$$

acting on the tensor product $H^{0} \otimes H^{1}$ of the representation space of a spinless massive representation of the Poincaré group tensored with a unitary representation of the homogeneous Lorentz group. The latter is precisely the setting for the Bros-Morschella localization on 3-dimensional de Sitter space which can be made explicit with the help of the Hörmander-Fourier transformation [42]. The De Sitter space localization arising from the second factor implies a spacelike directional localization in the $\mathrm{d}=1+3$ Minkowski spacetime which together with the pointlike localization coming from the first factor amounts to a localization along semiinfinite spacelike strings. The analytic mathematical details are somewhat more demanding as for massive representation and the reader is referred to the literature [11].

The associated string-localized field operators are defined on the Fock-space over the irreducible representation space and turn out to be of the form

$$
\begin{aligned}
& \Phi^{\alpha}(x, e)=\int_{\partial V^{+}} d \mu(p)\left\{e^{i p x} u^{\alpha}(p, e) \cdot a(p)+e^{-i p x} \overline{u^{\alpha}(p, e)} \cdot a^{*}(p)\right\} \\
& D_{\kappa}(R(\Lambda, p)) u^{\alpha}\left(\Lambda^{-1} p, e\right)=u^{\alpha}(p, \Lambda e) \\
& u^{\alpha}(p, e) \equiv e^{-i \pi \alpha / 2} \int d^{2} z e^{i k z}\left(B_{p} \xi(z) \cdot e\right)^{\alpha} \\
& \text { with } \xi(z)=\left(\frac{1}{2}\left(|z|^{2}+1\right), z_{1},-z_{2}, \frac{1}{2}\left(|z|^{2}-1\right)\right)
\end{aligned}
$$

where the intertwiner $u^{\alpha}$, which depend on a complex parameter $\alpha^{15}$ (and on $p$ via the boost $B_{p}$ ) are determined by the intertwining property in the second line and certain analyticity requirements (with a complex parameter $\alpha$ to be explained). The dot between the pre-factors $u^{\alpha}(p, e)$ and the creation and annihilation operators $a^{*}(p), a(p)$ (that depend also on $k \in \mathbb{R}^{2}$, suppressed by the notation) stands for integration over $k$ with respect to the measure $\delta\left(|k|^{2}-\kappa^{2}\right) d^{2} k$. The $k$-dependence of $u^{\alpha}, a$ and $a^{*}$ has thus been transferred to the dependence of the field $\Phi(x, e)$ on the space-like direction $e$. The field is an operator-valued distribution in $x$ and $e$. In more physical terms $\Phi(x, e)$ is a quantum field which fluctuated in the vacuum state in 4-dimensional Minkowski space as well in 3-dimensional de Sitter space (i.e. there is a quantum localization in both spaces [42]). It has the following properties that justify the terminology "stringlocalized":

\footnotetext{
${ }^{15}$ Although the Bros-Moschella setting fixes the parameter $\alpha$ to $\operatorname{Re} \alpha=-1$, the intertwiners which convert the Euclidean degrees of freedom into internal fluctuating string degrees of freedom exist (in the distribution theoretical) sense for all $\alpha$; in fact the continuous parameter $\alpha$ characterizes the linear part of the string Borchers class.
} 
- If $x+\mathbb{R}^{+} e$ and $x^{\prime}+\mathbb{R}^{+} e^{\prime}$ are space-like separated then

$$
\left[\Phi^{\alpha}(x, e), \Phi^{\alpha^{\prime}}\left(x^{\prime}, e^{\prime}\right)\right]=0
$$

while the commutator is nonzero for some $e, e^{\prime}$ if the endpoints only are space-like separated

- The transformation law of the field is consistent with this localization:

$$
U(a, \Lambda) \Phi^{\alpha}(x, e) U(a, \Lambda)^{-1}=\Phi^{\alpha}(\Lambda x+a, \Lambda e)
$$

- After smearing with compactly supported test functions in $x$ and $e$ (in certain $\alpha$-ranges a smearing in $e$ is unnecessary), the field operators generate a dense set in Fock space when applied to the vacuum vector $|0\rangle$. This is the appropriately string-adapted version of the Reeh-Schlieder [43] property which plays a crucial role in the mathematical physics literature on algebraic QFT.

The second statement is (as in the standard finite spin case) a result of the above intertwining properties of $u^{a}(p, e)$. This intertwiner function must have a certain complexity, since according to the first above property $\Phi$ must amalgamate Minkowski and De Sitter spacetime in such a way that the commutation properties are not simply those of a tensor product between two-point function in both spaces ${ }^{16}$ (which would have too strong commutation properties). For an explicit representation of $u^{a}(p, e)$ in terms of the Hörmander-Fourier transformation on de Sitter space we refer to [11].

Although it does not seem to be possible to express the string-localization intertwiner $u^{\alpha}$ and the resulting c-number commutator in terms of known functions, its existence and analytic properties can be proven from the representation. It turns out that the continuous parameter $\alpha$ (apart from the integer nonnegative values $0,1, .$.$) can be chosen at will and the different relatively lo-$ cal string fields in the same Fock space correspond to the linear part of the local equivalence (Borchers) class. They are the analogs of the discrete family of fields associated to the standard $(\mathrm{m}, \mathrm{s})$ Wigner representation by taking $u(p, s)$ and $v(p, s)$ intertwiners of increasing length $l \geq 2 s+1[44]$. Presently the important question of whether the operator algebra generated by these stringlocalized fields contains compactly localizable subalgebras (possibly generated by pointlike localized composite fields) is under investigation [45].

Although the same construction with $\alpha$-dependent intertwiners $u^{\alpha}(p, e)$ applies as well to the massive $(m, s)$ representation and leads to semiinfinite stringlocalized fields, their application to the vacuum generates the same one-particle Wigner representation spaces as the $e$-independent standard textbook intertwiners $u(p)$ which belong to pointlike fields. They could however play a role in enlarging the possibilities for interactions. Whereas the case of interacting

\footnotetext{
${ }^{16}$ In particular it cannot be reduced to standard type Jordan-Pauli type of zero mass commutators.
} 
pointlike localized fields [44] has been studied since the very beginning of field theory (and led to the result of renormalized perturbation theory in which the pointlike localization is maintained in every order), little is known about the interactions of string-localized fields.

There are indications that if one implements interactions with polynomials of string fields $A(x, e)$, the perturbation will maintain string stability in the sense that the resulting fields will not have a localization which spreads beyond the original string. This makes the string-like localization an excellent candidate for a type non-local theory which still maintains an extended form of causality. For zero mass finite spin representation the possibilities of pointlike free fields are more restricted than in the massive case. For example the Wigner representation for photons admit a description in terms in terms of pointlike field strength but not in terms of a pointlike vector potential. The only possibility for a physical (i.e. one without unphysical gauge-dependent degrees of freedom) vector potential is a string localized vector potential [45]. This suggests to use string interactions as an interesting alternative to gauge interactions.

Another type of string-localized field arises from the massive Wigner representation in $\mathrm{d}=1+2$ for (abelian) generic real values of the spin (different from (half)integer). Such generic "anyonic" values activate the rich covering structure of the 3-dimensional Lorentz group. The covering structure beyond the double covering requires to define real localization subspaces more carefully by attaching a spacelike direction $e$ such that $W+e \subset W[46]$. The directions $e$ are again points in a De Sitter space, but since this space is now two-dimensional this de Sitter space has an infinite covering. In this way the pairs $\tilde{W} \equiv(W, e)$ and finally also the sharpened pointlike data $(x, e)$ can be used to model a substitute for covering space of spacelike wedges respectively of spacelike strings which matches the Bargmann covering structure of the Lorentz group ${ }^{17}$. The geometric role of the spacelike directions is to have a substrate on which the center of the covering can act nontrivial. In this way the string nature of the resulting objects is already preempted by the covering structure of the modular wedge formalism which requires the definition of a reference wedge from which the "winding number" is counted. One easily shows that unlike the (half)integer spin case there is now a complex phase factor between the symplectic complement of $H_{r}(\tilde{W}), \tilde{W}:=(W, e)$ and its geometric complement i.e. $H_{r}(\tilde{W})^{\prime}=$ Phase $\cdot H_{r}\left(\tilde{W}^{\prime}\right)$. Since the symplectic complement is the one-particle projection of the von Neumann commutant and the geometric complement is given by the square root of the $2 \pi$ spin rotation, the phase is obviously related to the spin-statistics phase of abelian braid group commutation relations (anyons). The presence of string localization can be analytically confirmed by showing the complex phase requires the triviality of compactly localized sub-

\footnotetext{
${ }^{17}$ Another way to associate a covering space with the Minkowski spacetime is to compactify Minkowski space and then take its universal covering. The symmetry group of the covering is the the covering of the conformal extension of the Poincaré group. the presence of covering groups beyond matrix groups implies the presence of interactions (see third section). The timelike covering of the compactification does not lead to strings but rather to a timelike braid group structure.
} 
spaces. The smallest intersections of wedges which are consistent with $\mathcal{C}+e \subset \mathcal{C}$ are spacelike cones with arbitrary opening angles.

This kind of string (referring to the "would be" string-like generators of the spacelike cone localized field algebras) is very different from the previous one since it owes its string-like nature not to the presence of an internal structure of the little group with internal string degrees of freedom. Fields transforming according to higher (than two-fold) coverings of $S O(2,1)$ carry no more degrees of freedom than Bosons or Fermions. Whereas the anyonic string resembles what one expects from Mandelstam strings (the oldest use of gauge invariant strings in QED is due to Jordan [48]) i.e. strings in gauge theories ${ }^{18}$, the strings with the internal degrees of freedom in the form of an infinite helicity tower are in some sense more like the objects of string theory (see however the caveats below). The anyonic strings are for various reasons (e.g. potential applications in solid state physics) of more immediate physical interests than the previous zero mass infinite spin strings, but their highly nontrivial field theory (there are no on-shell free fields) will not be considered here.

String-localized objects are radically different from the properties of quantized Nambu-Goto strings through which Polyakov found the string interpretation of the dual model in terms of functional integrals. There exist two different quantizations of the N-G string. The more intrinsic approach is due to Pohlmeyer [49] and consists in extracting a complete set of classical invariants (called Pohlmeyer charges) which together with the generators of the Poincaré group form a closed Poisson algebra. This task has been almost completed, and in this way the system was identified with an integrable system in the classical sense. The quantization of this algebra ${ }^{19}$, i.e. the search for an algebra of operators which have commutation relations which mimic the Poisson structure as much as possible, is still an unfinished problem. The Pohlmeyer strings are not string-localized, but their algebra of invariants share with the string-localized fields that they exist as Poincaré covariant theories in all spacetime dimensions $d \geq 3$ i.e. there is no mysterious distinction (resulting from the non-intrinsic canonical quantization) of $d=10,26$.

The other approach is that which led to string theory proper and consists in quantizing before eliminating the constraints and afterwards taking care of the constraints in the spirit of the BRST cohomological approach. The localization aspect was investigated [50][51] and it was found that their quantum localization defined in terms of commutators is pointlike, unlike its classical appearance. There exist also recent arguments that the S-matrix of string field theory can be associated with a (possible infinite) family of local fields [52].

Since the quantization of a classical Lagrangian in its quantization should not lead to two different QT, this raises the question whether there is a right

\footnotetext{
${ }^{18}$ There are also the semiinfinite Buchholz-Fredenhagen strings [47] of local quantum physics coming from the classification of admissible superselection charges in the presence of a mass gap. The authors believe that they are the model independent and rigorous version of strings in massive gauge theories with confinement.

${ }^{19} \mathrm{It}$ is believed that the correct quantum theory for integrable systems is obtained by quantizing its invariant charges.
} 
one and which is it. From the viewpoint of quantization of integrable systems it is Pohlmeyer's quantization; however the string theoretic reading of the dual model requires the use of the canonical quantization and the BRST formalism.

\section{Wedge-localized PFGs}

Non-local auxiliary operators play a pivotal role in nonperturbative (and nonLagrangian) constructions of QFT models. Their purpose is to generate operator algebras which are localized in extended causally closed regions. The best studied case is that of wedge-localized generators without vacuum polarization, which lead among other things to the $\mathrm{d}=1+1$ Zamolodchikov-Faddeev algebras of factorizing models.

Let us briefly look at this interesting new aspects by explaining the meaning of vacuum-polarization-free generators shortly called PFGs. Let $\mathcal{O}$ be a causally closed, simply connected region in Minkowski spacetime, typically (in order of decreasing size) a wedge $\mathcal{W}$, a spacelike cone $\mathcal{C}$ or a compact double cone $\mathcal{D}$. A $\mathcal{O}$-associated PFG $F$ is a not necessarily bounded operator affiliated with the operator algebra $\mathcal{A}(\mathcal{O})$ (denoted by $F \eta \mathcal{A}(\mathcal{O})$ ) such that the vector obtained by applying $F$ once to the vacuum $F \Omega$ contains only a one-particle component i.e. has no vacuum multiparticle polarization parts. The operator algebras of a free field theory clearly possess PFGs for every spacetime region. However wedge-localized PFGs even exist in interacting theories (to the extend that have isolated one-particle states). This is a general consequence of modular theory. But in most cases these PFG are not useful because their translates do not admit Fourier transforms and their extremely bad domain properties prevent their successive applications as in Wightman field theory.

The useful PFG are the so-called "tempered PFG". They generally do not exist in the presence of interactions, even if we allow the region to be as large as a wedge. In fact if a PFG $F$ exists for a subwedge region (like a spacelike cone $C$ or a double cone $\mathcal{D}$ ), then it follows that the $F$ is in fact a smeared free field (with support of the test function in the localization region). The analytic part of the proof is very similar to the proof of the Jost-Schroer theorem [43], the slight complication due to the fact that the operator $F$ has a prescribed localization property but no simple (tensorial) covariance behavior is easy to account for.

So the non-existence of subwedge PFG is the precise intrinsic local characterization for the presence of interactions which turns out to be equivalent (under the assumption of validity of the crossing property of formfactors) to $S_{\text {scat }}=1$. Modular theory establishes the existence of wedge-localized PFGs, but PFGs in the stronger sense of temperateness only exist in case of interactions without real (on-shell) creation and annihilation in scattering processes. It turns out that a purely elastic $S_{\text {scat }}$ are only possible in $\mathrm{d}=1+1$ and it is believed that this possibility is exhausted by the family of factorizing models. Schematically, limiting the notation to the simplest case of a scalar scattering matrix in a factorizing theory involving one particle only (e.g. the Sinh-Gordon model) we 
have for the wedge localized PFG $A(x)$ the following on-shell representation [20]

$$
\begin{aligned}
A(x) & =\int\left(Z(\theta) e^{i p(\theta) x(\chi)}+\text { h.c. }\right) d \theta \\
p(\theta) & =m(\operatorname{ch} \theta, \operatorname{sh} \theta), x(\chi)=r(\operatorname{sh} \theta, \operatorname{ch} \theta)
\end{aligned}
$$

where the second line defines the mass shell rapidity parametrization as well that of the right wedge. The $Z^{\#} s$ are not the standard creation/annihilation operators, but they rather fulfill the Zamolodchikov-Faddeev algebra relations:

$$
\begin{aligned}
Z(\theta) Z^{*}\left(\theta^{\prime}\right) & =S\left(\theta-\theta^{\prime}\right) Z^{*}\left(\theta^{\prime}\right) Z(\theta)+\delta\left(\theta-\theta^{\prime}\right) 2 \\
Z(\theta) Z\left(\theta^{\prime}\right) & =S\left(\theta^{\prime}-\theta\right) Z\left(\theta^{\prime}\right) Z(\theta)
\end{aligned}
$$

Therefore $A(x)$ can not be a local field (except $S(\theta)= \pm 1$ ). Some application of modular theory of operator algebras for the wedge region shows that

$$
\begin{aligned}
A(f) & =\int A(x) f(x) d^{2} x, \sup p f \subset W \\
\mathcal{A}(W) & =\operatorname{alg}\{A(f), \sup p f \subset W\}
\end{aligned}
$$

generate the wedge algebra.

In contradistinction to pointlike localized fields the sharpening of the support of $f$ to a subregion inside $W$ does not help since the localization region of resulting operator does not follow the classical picture of the support of $f$. The only way to restrict the localization is to define algebras with the more restricted localization by forming the intersections of wedge algebras which contain $D$ i.e.

$$
\mathcal{A}(D)=\cap_{W \supset D} \mathcal{A}(W)
$$

which for the case of $d=1+1$ at hand can be shown to reduce to

$$
\begin{aligned}
\mathcal{A}(D) & =\mathcal{A}(W) \cap \mathcal{A}\left(W_{a}\right)^{\prime} \\
D & =W \cap W_{a}^{\prime}
\end{aligned}
$$

the condensed notation requires some explanation. $W_{a}$ is the result of applying a right spacelike translation by $a$ to $W$ and the upper dash on regions denotes their spacelike complement which in the case of a wedge is just the opposite left wedge. The same dash but on operator algebras denotes the von Neumann commutant in the ambient Hilbert space. The computation of intersections of von Neumann algebras is a complicated problem for which no general methods exist. However if the generators of wedge algebras have a fairly simple structure as in our case, one can solve the characterizing relations for the desired $\mathcal{A}(D)$ affiliated operators $A$ as those which obey the relation

$$
A \subset A(W) \text { s.t. }\left[A, U(a) A(f) U(a)^{-1}\right]=0
$$

In the spirit of the old LSZ formalism one can then make an Ansatz in form of a power series in $Z(\theta)$ and $Z^{*}(\theta) \equiv Z(\theta-i \pi)$ (corresponding to the power 
series in the incoming free field in LSZ theory)

$$
A=\sum \frac{1}{n !} \int_{C} \ldots \int_{C} a_{n}\left(\theta_{1}, \ldots \theta_{n}\right): Z\left(\theta_{1}\right) \ldots Z\left(\theta_{n}\right)
$$

Each integration path $C$ extends over the upper and lower part of the rim of the $(0,-i \pi)$ strip. The strip-analyticity of the coefficient functions $a_{n}$ expresses the wedge-localization of $A$. It is easy to see that these coefficients are identical to the vacuum polarization form factors of $A$

$$
\left\langle\Omega|A| p_{n}, . . p_{1}\right\rangle^{i n}=a_{n}\left(\theta_{1}, \ldots \theta_{n}\right)
$$

whereas the crossing of some of the particles into the left hand bra state leads to the connected part of the formfactors

$$
{ }^{\text {out }}\left\langle p_{1}, . . p_{l}|A| p_{n}, . . p_{l+1}\right\rangle_{\text {conn }}^{\text {in }}=a_{n}\left(\theta_{1}+i \pi, \ldots \theta_{l}+i \pi, \theta_{l+1}, . . \theta_{n}\right)
$$

The algebraic identity (24) involving the in $Z$ linear generator $U(a) A(f) U(a)^{-1}$ relates the $a_{n}$ coefficients whose $\mathrm{n}$ differs by 2 . This is nothing else than the famous "kinematical pole condition" first introduced as one of the construction recipes by Smirnov [53]. The solutions together with the Payley-Wiener meromorphic characterization of the size of $D$ defines a space of formfactors or $A^{\prime} s$ in the sense of bilinear forms.

If one wants $A$ to have simple covariance properties one should think of a basis of pointlike fields (the generalization of Wick monomials to the realm of interaction) which in this context does not create short distance problems since formfactors by definition do not contain short distance fluctuations. Hence if we would know that the intersected algebras $\mathcal{A}(D)$ are nontrivial then the present approach serves as a calculational tool for the formfactors of these operators. Direct calculations of correlation functions with the aim of showing the existence via a GNS kind of reconstruction theorem failed because one has not been able to control the sum over intermediate particle states which formally links correlation functions of products of operators with formfactors of these operators.

In this situation it is interesting to note that there has been a recent proposal by Buchholz and Lechner to use powerful modular methods of operator algebras ("modular nuclearity") instead if handling infinite intermediate state sums using formfactors. If this modular nuclearity property can be checked for the wedge algebras than the nontriviality of the intersection is guarantied and one would have succeeded to proof the existence of a large class of nontrivial models whose fate would otherwise fall under the spell of short distance properties.

The idea in this form depends on the existence of nontrivial wedge-localized PFG (only possible in absence of inelastic scattering processes) and can not be generalized to non factorizing models or to higher dimensions. Since the presentation of some highly speculative ideas how to get around these problems would lead to far away from the main subject of this paper we refer to forthcoming work.

On the other hand Brunetti Guido and Longo [41] constructed the family of wedge-localized real subspaces of the Wigner representation spaces by 
combining purely group theoretical ideas with aspects of modular theory. Although the subwedge (spacelike cones, double cones i.e. the natural simply connects causally complete regions obtained by intersecting wedges) localized real subspaces have no geometric modular characterization, they can easily be constructed by intersecting wedge-localized spaces. The work of these authors culminated in a theorem that positive energy representations of the Poincaré group always have nontrivial spacelike cone-localized subspaces for arbitrary small opening angles of the spacelike cone and that the wedge like localization can be re-obtained by additivity from spacelike cone localized subspaces. In case of (half)integer spin representations one can show that the compact spacelike cone intersections are nontrivial by constructing a dense set of double cone localized wave functions. However there were two class of representations which did not allow compact localization (i.e. the double cone localization spaces were trivial), the $\mathrm{d}=1+2$ representations with "anyonic" spin ( $\mathrm{s} \neq$ (half)integer) and the famous Wigner family of zero mass infinite helicity representations. Both cases lead to nontrivial spacelike cone localized subspaces and hence it was suspected that these representations may be associated with string-localized field theories. This turns out to be true; but only in the infinite spin case the stringlocalized fields are genuine free fields, whereas in the anyonic case the string localized fields applied to the vacuum are always accompanied by vacuum polarization ${ }^{20}$ which throws them off mass shell.

The modular approach to the $d=1+1$ factorizing models is very similar in that the principle structure is always associated with wedge-localized algebras and the subwedge-localized objects are formed by intersections. The main difference is that in all situations of positive energy Wigner representations (with the exception of the case of $\mathrm{d}=1+2$ anyons) there also exist subwedge-localized PFGs and their associated algebras are obtained in a very simple functorial way by from a spatial modular theory in a functorial way (the CCR or CAR functor). This had led me already in a very early stage of my investigations [54][55] to view the whole constructive program based on modular theory as an extension of the functorial relation between spatially and algebraically localized objects to the realm of interactions.

In this section I have been using two different looking, but nevertheless equivalent intrinsic definitions of the meaning of "interacting". On the one hand one can take the absence of PFGs associated to subwedge-localized algebras as the local definition of absence of interactions (and their presence in the opposite situation). On the other hand there exists the global definition in terms of the triviality of the S-matrix i.e. $\mathrm{S}=1$. The modular aspects of wedge-localized algebras permits to identify these two definitions.

\footnotetext{
${ }^{20}$ Only wedge-localized algebras of anyon theories support generators (tempered PFGs [13]) without vacuum polarization which can be viewed as being directly associated with wave functions from the the corresponding wedge-localized Wigner representation subspaces.
} 


\section{Non-local aspects of QFT in noncommutative Minkowski spacetime}

More than a decade ago Doplicher, Fredenhagen and Roberts [14] started to investigate the feasibility of a QFT on noncommutative Minkowski space. Their original motivation was to study implications of certain uncertainty relations which arose from limitations on measurements in small volumes if one requires that no black hole horizons should be created by performing such measurements alone i.e. without doing anything else. They then realized that in case one ignores curvature these uncertainty relations can be saturated in terms of a noncommutative Minkowski spacetime i.e. an algebra affiliated with hermitian localization operators which fulfill (in a suitable normalization)

$$
\begin{aligned}
{\left[q_{\mu}, q_{\nu}\right] } & =i \lambda_{P}^{2} Q_{\mu \nu},\left[q_{\lambda}, Q_{\mu \nu}\right]=0 \\
Q_{\mu \nu} Q^{\mu \nu} & =0,\left(\frac{1}{16} Q_{\mu \nu} \widetilde{Q}^{\mu \nu}\right)^{2}=1
\end{aligned}
$$

where the matrix $Q$ is central-valued and transforms under L-transformation like an antisymmetric tensor and $\widetilde{Q}$ the associated pseudo tensor. The "Planck length" $\lambda_{P}$ is a parameter whose intended role is that for large distances the spacetime is to return to Minkowski spacetime.

An irreducible representation of the $q^{\prime} s$ would require numerical values $\sigma$ of $Q$ which would destroy L-invariance. The L-transformed $q^{\prime}=\Lambda q$ leads to another irreducible representation with another commutator matrix $\sigma^{\prime}$ which results from L-transforming the antisymmetric tensor $\sigma_{\mu \nu}$. The minimal *algebra on which the Poincaré transformations can act as automorphisms is therefore a direct integral over $\Lambda q$ with the Haar measure of the L-group. Since the Lorentz group only enters the algebraic structure via its transitive action on the $\sigma_{\mu \nu}$ matrices, the direct integral actually involves a measure on $\Sigma$ which is induced by the Haar measure of the L-group. It is comforting to know that this formal *algebra on which the Poincaré group acts as an automorphism can be elevated to a mathematically respectable $C^{*}$-algebra whose regular representation is unique (up to quasiequivalence) [14]. The spectral values which the central elements $Q$ take consists of all values $\sigma \in \Sigma$ with the above restriction for the matrix entries; explicitly this set $\Sigma$ turns out to be isomorphic to the 4-dimensional tangent space of a sphere extended by a reflection $\Sigma \simeq T S^{2} \times\{-1,1\}$.

There exist Poincaré covariant representations of the algebra affiliated with these commutation relations and the full spectrum $\Sigma$ (which simply consists of the space of numerical matrices fulfilling the above algebraic conditions (28)) [14] of the nontrivial center; this situation is summarily referred a as the noncommutative Minkowski spacetime $M_{q u}$. The representation of the translations (incorporation of the momentum space) requires to tensor the Hilbert space $H$ which carries the $q$-representation with its conjugate $H_{r e p}=H \times \bar{H}$. Different from the positive energy representations featuring in particle physics, the translational part is not related to support properties in momentum space (in particular no positive energy restriction). 
What is meant by a field on such a noncommutative Minkowski spacetime can be best explained in case of a free field ${ }^{21}$. Formally it is obtained by substituting the spacetime variable by the above operator $q$

$$
\begin{aligned}
A(q) & =\int\left(e^{-i p q} a(p)+h . c .\right) \frac{d^{3} p}{2 p_{0}} \\
H & =H_{\text {rep }} \otimes H_{F o c k}
\end{aligned}
$$

where the second line denotes the Hilbert space of the free model and the first factor is the previously explained covariant representation space. The Poincaré transformations do not only act on the particle variables (including the transformations of their "non-commutative positions"), but they also act on the central operators $Q$ and transform those parts of their spectrum together the localizing wave functions. In this way we clearly keep the Wigner particle picture as one of the pillars of any relativistic particle theory. If we want to return to a Fock space description, we have to take the (partial) expectation value in a state $\omega$ on the localization space $M_{q u}$ which leads to expressions of the form [14][22] e.g.

$$
\begin{aligned}
& \omega \rightarrow \omega\left(A\left(q+x_{1}\right) \ldots A\left(q+x_{n}\right)\right)= \\
& =\int f_{\omega}\left(x_{1}, x_{2}, \ldots x_{n} ; \xi_{1}, \xi_{2}, \ldots \xi_{n}\right) A\left(\xi_{1}\right) A\left(\xi_{2}\right) \ldots . A\left(\xi_{n}\right) d \xi_{1} d \xi_{2} \ldots d \xi_{n}
\end{aligned}
$$

This leaves us with smeared non-local usual operator in Fock space (written in terms of a non-local monomial of local free fields) where the non-locality comes from collecting the product of $q$-exponentials in one exponential only ${ }^{22}$ on which the state $\omega$ over the $q$-algebra may be evaluated. If one starts e.g. with an $\omega$ corresponding to "minimal localization" [14], the spectral values of $Q$ are restricted to a compact submanifold $\Sigma_{1}$ which is left invariant by the rotation and translation subgroups whereas a L-boost will change its position within $\Sigma$. Since L-invariant states on $M_{q u}$ do not exist, the field theoretic expressions on $H_{F o c k}$ are even before test-function smearing already delocalized and noncovariant (in the sense that they are not fitting the tensor/spinor calculus of Lagrangian quantization). The commutator of two such fields evaluated on $\Sigma_{1}$ associated minimal states $\omega$ exhibits a Gaussian decrease in spacelike direction. This is faster than the Yukawa decrease allowed by the Borchers-Pohlmeyer theorem mentioned in the introduction, but one has to keep in mind that a free field theory on $M_{q u}$ is quite different from a Wightman theory on ordinary Minkowski spacetime $M$ so that the analyticity properties used in the derivation of the Borchers-Pohlmeyer theorem are violated.

Although there is no problem with free noncommutative QFT, the interacting situation is still in a very precarious state. The problem with interactions

\footnotetext{
${ }^{21}$ This restriction is not only taken for pedagogical reasons; it is presently not quite clear whether the standard implementation of interactions in terms of polynomials of (noncommutative) field products is appropriate.

${ }^{22}$ The non-commutativity of the $q$-exponentials converts the ordinary product of field operators into a "twisted" product. The twist factors are center-valued and it is only their evaluation in a state $\omega$ on the $M_{q u}$ which leads to $\mathbb{C}$-valued weighted integrals over the $\vec{Q}$-spectrum.
} 
is similar to those problems on which the old attempts (mentioned in the introduction) failed.

The indispensable property which any particle physics theory hs to deliver, whether P-covariant and non-local in the old sense or P-covariant and non-local in the new sense of non-commutative Minkowski spacetime, is a P-invariant S-matrix which describes scattering between Wigner multi-particle in and out states.

Before one tries to formulate this requirement in terms of new interacting fields on $M_{q u}$ in a LSZ setting of asymptotic convergence, one may try to make mathematical experiments with a Gell-Man-Low like Ansatz for an S-matrix or similar attempts with formulas close to the standard ones. The results in this direction have been only partially successful. On the one hand it was possible to overcome previous difficulties with unitarity and ultraviolet divergencies [21]. These perturbative calculations can even be arranged in such a way that Feynman graphs supplemented with other rules continue to be useful [23]. On the other hand it is presently not known of how to obtain an S-matrix in the aforementioned intrinsic sense of particle physics. It seems that the intuitive idea that noncommutative Minkowski space asymptotically (for large distances) turns into standard Minkowski spacetime does not work for on-shell quantities like the S-matrix whose definition should be protected against short distances.

Note that in the C-P direct particle interaction theory the non-locality also prevented the existence of covariant tensor objects corresponding to Heisenberg field ${ }^{23}$. But thanks to the scattering equivalences this, did not spoil the existence of a clustering unitary S-matrix. The D-F-R model is still best by the problem whether interacting correlation functions in $\omega \times \omega_{\text {vac }}$ states will fulfill cluster fall-off properties, which is a prerequisite for the existence of a clustering S-matrix.

On the other hand one should perhaps not put too much faith in the particular D-F-R model of fields on $M_{q u}$ but investigate more profoundly other implementations of their physically well-motivated commutation relations. Perhaps there is also a message to be drawn from the successful modular localization setting. The representation of the Poincaré-group (including reflections) on $H_{r e p} \times H_{W i g}$ (where $H_{W i g}$ stands for the Wigner irreducible particle representation) has also a wedge-associated Tomita operator and therefore is subject to the setting of modular localization (although with less restrictive properties since the representation in the first factor is not of positive energy [41]) which seems to allow for a wider starting point than that defining the D-F-R model.

\section{Concluding remarks, outlook}

In this work we presented post renormalization attempts to formulate theories in particle physics which either go beyond the frontiers of relativistic locality

\footnotetext{
${ }^{23}$ The theory is not capable to produce formfactors i.e. particle matrix elements of covariant currents etc.
} 
(spacelike locality and timelike causality) but still maintaining Wigner's particle concept, or proposals which aim at local theories but use non-pointlike operators in intermediate steps as an essential constructive tool.

Since the word "non-local" is as non-revealing as a "non-elephant", one needs to specify in what sense they non-local theories or non-local operators deviate from locality.

This is most easy done in case of the wedge-localized objects (section 4). Whereas Wigner particle creation/annihilation operators are non-local in the sense that they can only be associated to the global algebra on all of Minkowski spacetime $^{24}$, wedge localized one particle states can already be obtained by applying specific operators (FPGs) associated with wedge-localized algebras to the vacuum. For subwedge regions this is not possible in the presence of interactions, so that regions whose causal closure is wedge-shaped are distinguished by presenting the best compromise between the de-localizing tendency of interacting particles and the locality principle which is historically (Faraday, Maxwell) associated with fields. Presently the constructive use of such wedgelocalized PFGs (whose existence is guarantied by the Tomita-Takesaki modular theory) is restricted to tempered PFGs [13], which turn out to be related to the Zamolodchikov-Faddeev algebraic structure. The attractive aspect of this intrinsic algebraic bootstrap formfactor approach [55][56] for mathematical physics is that the age old problem of existence of nontrivial QFTs becomes separated for the first time from the issue of ultraviolet behavior and instead passes to properties which refer phase-space degrees of freedom associated with wedge-localized algebras. In $\mathrm{d}=1+1$ this amounts to check their "nuclear modularity" property [57].

The non-local "direct particle interaction" approach of Coester and Polyzou (section 2) has its point of departure in the extreme opposite setting of relativistic quantum mechanics i.e. it is a framework without any vacuum polarization structure. The only conceptually somewhat unusual aspect of this approach is the two-step way in which the inductive (Bakamijan-Thomas) construction of a Poincaré covariant but non-cluster-factorizing n-particle system is followed by the enforcement of clustering via (Sokolov) scattering equivalences. Although it was not our aim to present this direct particle approach as a serious contender for a fundamental non-local interactions, we think that it should be part of the intellectual preparation for somebody who wants to study the issue of non-local interactions to be aware that the reason why this possibility was missed in the history of the pre-renormalization particle physics was precisely that non-locality and the field-based formalism of QFT do not mix well.

Another advantage of contrasting the aforementioned algebraic approach to QFT via wedge algebra generators with the direct particle interaction setting is a possible deeper understanding of vacuum polarization as the most characteristic property of local QFT. Since the days when Heisenberg discovered that near the surface of spatial localization regions of "partial" charges there

\footnotetext{
24 "Almost local" operators [7] i.e. operators which are "essentially" localized in a finite region but have "tails" extending to spatial infinity are not considered here.
} 
are always (infinitely big for sharp surfaces) vacuum polarization clouds (even without interactions), and ever by since the ancient observation Furry and Oppenheimer that interactions implemented by Lagrangian perturbations always lead to field-states (states obtained from the vacuum by the application of fields) whose one-particle contribution are inexorably accompanied by vacuum polarization clouds, it became desirable to have a more intrinsic quantitative nonperturbative understanding of these phenomena. Important effects as the on-shell crossing property and the localization-induced thermal effects (Hawking-Unruh radiation from causal Rindler horizons, area proportionality of localization induced entropy etc.) depend on a better understanding of vacuum polarization.

The non-locality behind the string-localization (section 3) has a clear historical motivation. Far from being a mere invention, it was looming there ever since Wigner wrote his famous article on the classification of irreducible representations of the Poincaré-group. But whereas for all the finite halfinteger spin representations there was a description in terms of local Euler-Lagrange setting following the prior rules of Jordan's field quantization, the Wigner zero mass infinite helicity representation turned out to be outside this historically cherished framework. This is the reason why the associated string-localized operator theory was discovered only recently; without any classical guide one needed a good understanding of modular localization before one could solve this problem.

The open question is whether in addition to filling a historical loophole, string localization can lead to new physics. The hope that this turns out to be the case is founded on the observation that there exist string-localized massive free fields $A(x, e)$ with nice properties which, as the zero mass infinite spin fields, live simultaneously on Minkowski spacetime (labeled by $x$ ) as well as on one dimensional lower de Sitter manifold of spacelike directions (labeled by $e$ ). One nice property which emerges from a study of their two-point function is that they are less singular in $e$-direction than e.g. the associated pointlike fields [58]. There is some hope that these objects may facilitate a perturbative construction of massive Buchholz-Fredenhagen strings whose existence is strongly suggested by methods of algebraic QFT [47], but for which no convincing example has been constructed yet.

The more popular incursions into the non-local realm are the various attempts to formulate and apply non-local QFT. Whereas the older attempts (see introduction) in the aftermath of renormalization theory were motivated by the avoidance of ultraviolet divergencies and the desire to extend Lagrangian quantization in order to incorporate particle formfactors as fundamental concepts, the more recent approaches (section 5) aim at non-locality via non-commutative modifications of Minkowski space. The motivation for this is twofold, on the one hand there are good physical reasons to look at the conceptual modifications of spacetime if one subjects the Einstein gravitational equations to the kind of quasiclassical requirements which in the context of the Maxwell equations in conjunction with quantum matter led Bohr and Rosenfeld to their uncertainty relations for electromagnetic field strength. In this spirit spacetime commutation were first derived in [14]. A more formal argument in the same direction but with a buildt-in violation of Lorentz invariance (a kind of quantum ether) 
came from string theory [59].

While the original purpose behind these proposals was to contribute ideas towards quantum gravity, their present use seems to be limited to QFT in noncommutative Minkowski spacetime. Although as non-local models of particle physics some of these attempts are mathematically much more sophisticated than the pedestrian old attempts via formfactor modifications of Lagrangians, they still must pass those requirements as the cluster factorization property and scattering theory on which the old attempts failed.

There has been a recent proposal to reconcile ideas of noncommutative geometry with the principle of local covariance [60]. The reason why noncommutative geometry cannot directly be applied to particle physics is that it is modelled on Euclidean space, and apart from very special situations one meets in Lagrangian quantization there is no analytic bridge between Euclidean and real spacetime. What makes this idea attractive is that not only do the authors propose to overcome the restrictions to euclidean spectral triples, but they seem to be the first who take the recently discovered principle of "local covariance" [61] serious and try to adapt it to a noncommutative context towards quantum gravity.

Acknowledgments: I am indebted to Fritz Coester and Wayne Polyzou as well as Dorothea Bahns and Gherardo Piacitelli for an interesting correspondence. To Karl-Henning Rehren I owe thanks for a critical reading of the manuscript.

\section{References}

[1] P. Christensen and C. Møller, On a convergent meson theory, Dan. mat. Fys. Medd.27, No.7 (1952) 1

[2] C. Bloch, On field theories with non-localized interactions, Dan. mat. Fys. Medd.27, No.8 (1952) 2

[3] C. Hayashi, Prog. Theor. Phys. 10, (1953) 533 and 11, (1954) 226

[4] See reviews by Kallen and Lehmann for the Mathematisches Zentralblatt, http://www.emis.de/cgi-bin/Zarchive?an=0049.27704

[5] Proceedings of the Conference of Theoretical Physics in Kyoto and Tokyo, Japan, Sept. 1953, ed. I. Imai, Nippon Bunka Iusatsusha (1954)

[6] R. Marnelius, Phys. Rev. D 19 (1974) 3411

[7] R. Haag, Local Quantum Physics, Springer 1996

[8] H.-J. Borchers and K. Pohlmeyer, Commun. Math. Phys.

[9] G. Marques and J. A. Swieca, Nucl. Phys. B43, (1972) 205

[10] F. Coester, Helv. Physica Acta 38, (1965) 7 
[11] J. Mund, B. Schroer and J. Yngvason, String-localized quantum fields from Wigner representations, math-ph/0402043

[12] S. Åks, Journ. Math. Phys. 6, (1965) 516

[13] H.J. Borchers, D. Buchholz and B. Schroer, Commun. Math. Phys. 219, (2001) 125, hep-th/0003243

[14] S. Doplicher, K. Fredenhagen and J. E. Roberts, Commun. Math. Phys. 172, (1995) 187

[15] C. Mead, Phys. Rev. II. Ser. 135, (1964) B 849

[16] H. S. Snyder, Phys. Rev. 71, (1947) 38

[17] T. D. Newton and E. P. Wigner, Rev. Mod. Phys. 21, (1949) 400

[18] L. Alvarez-Gaume and M. A. Vazquez-Mozo, General properties of noncommutative field theories, hep-th/0305093

[19] M. Chaichian et al., Towards an axiomatic formulation of non-commutative QFT, hep-th/0402212

[20] B. Schroer, Constructive proposals for QFT based on the crossing property and on lightfront holography, hep-th/0406016

[21] D. Bahns, S. Doplicher, K. Fredenhagen and G. Piaticelli, Ultraviolet-finite quantum field theory on quantum spacetime, Commun. Math. Phys. 237, (2003) 221, hep-th/0301100

[22] D. Bahns, The ultraviolet-finite Hamiltonian approach on the noncommutative Minkowski space, hep-th/0401219

[23] G. Piacitelli, Non local theories, new rules for old diagrams, hep-th/0403055

[24] B. Bakamjian and L. H. Thomas, Phys. Rev. 92, (1953) 1300

[25] F. Coester and W. N. Polyzou, Phys. Rev. D 26, (1982) 1348 and references therein

[26] W. N. Polyzou, Nucl-th/0201013, J. Math. Phys.

[27] N. S. Sokolov, Doklady Akad. Nauk USSR 233, (1977) 575

[28] W. N. Polyzou, Ann. Phys. 193, (1989) 367

[29] B. Kuckert, SpinESTtatistics in Non-relativistic Quantum Mechanics, quant$\mathrm{ph} / 0208151$

[30] H.P. Halvorson, Philosophy of Sciences 68, (2001) 111

[31] D. Malament, in Perspectives on Quantum Reality, ed. R. Clifton Dortrecht Kluwer (1996) 1 
[32] G. C. Hegerfeldt, Phys. Rev. Lett. 72, (1994) 596

[33] D. Buchholz and J. Yngvason, Phys.Rev.Lett. 73 (1994) 613

[34] J. Yngvason, The role of type III factors in quantum field theory, math$\mathrm{ph} / 0411058$

[35] J. Bros and D. Buchholz, Nucl. Phys. B 627, (2002) 219

[36] B. Schroer and H-W. Wiesbrock, Rev. Math. Phys. Vol. 12, No.3, (2000) 461

[37] D. Guido and R. Longo, Annales Henri Poincare 4 (2003) 1169-1218, grqc/0212025

[38] R. M. Wald, Quantum field theory in curved space time and black hole thermodynamics, (1994) University of Chicago Press

[39] H. P. Halvorson and R. K. Clifton, British Journal for the Philosophy of Science

[40] J. Yngvason, Commun. Math. Phys. 18, (1970) 195

[41] R. Brunetti, D. Guido and R. Longo, Rev. Math. Phys. 14, (2002) 759

[42] J. Bros and U. Moschella, Rev. Math. Phys. 8, (1996) 324

[43] R. F. Streater and A. S. Wightman, PCT SpinEStatistics and All That, Benjamin 1964

[44] S. Weinberg, The Quantum Theory of Fields I, Cambridge University Press 1995

[45] J. Mund, B. Schroer and J. Yngvason, in preparation

[46] J. Mund. J. Math. Phys. 44, (2003) 2037

[47] Buchholz and Fredenhagen. Commun. Math. Phys. 84, (1982) 1

[48] P. Jordan, Zeitschrift fuer Physik 95, (1935) 202

[49] K. Pohlmeyer, hep-th/9805057, Annalen der Physik 8, (1999)

[50] E. Martinec, Class. Quant. Grav. 10, (1993) L187

[51] J. Dimock, J. Math. Phys. 41, (2000) 40

[52] D. G Erler and D. J. Gross, Locality, Causality and an Initial Value Formulation of open Bosonic String Field Theory, hep-th/0409179

[53] F. A. Smirnov, Advanced Series in Mathematical Physics 14, World Scientific (1992) 
[54] B. Schroer, Nucl. Phys.B. 499, 537 (1997)

[55] B. Schroer, Annals of Physics 295, (1995) 190

[56] G. Lechner, Lett. Math. Phys. 64, (2003) 137

[57] D. Buchholz and G. Lechner, Modular Nuclearity and Localization, math$\mathrm{ph} / 0402072$

[58] J. Mund, B. Schroer and J. Yngvason, in preparation

[59] N. Seiberg and E. Witten, J. High Energy Phys. 09, (1999) 032

[60] and R. Verch, Local covariant quantum field theory over spectral geometries, gr-qc/0405057

[61] R. Brunetti, K. Fredenhagen and R. Verch, Commun. Math. Phys. 237, (2003) 31 